\title{
Long-term dabigatran therapy reduces the risk of recurrent venous thromboembolism
}

Effective use of warfarin requires frequent monitoring. Patients treated with dabigatran, a novel oral anticoagulant used to prevent venous thromboembolism (VTE) after joint replacement or to prevent stroke related to atrial fibrillation, do not need to be monitored as often. "Physicians stop anticoagulation after typically 6 months in patients with VTE, despite the residual risk for recurrence," explains Sam Schulman, lead investigator of two new trials in which long-term dabigatran therapy was investigated. He cites "inconvenience and bleeding risk of anticoagulant treatment with warfarin" as the primary reasons that physicians stop anticoagulation therapy. Schulman and colleagues, therefore, compared the safety and efficacy of dabigatran with either warfarin or
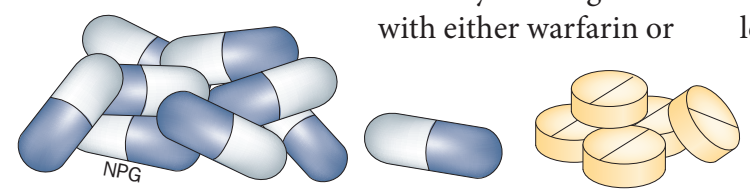

placebo in the double-blind, randomized RE-MEDY and RE-SONATE trials, respectively. Dabigatran was effective in preventing recurrent VTE, and was associated with a lower risk of bleeding than warfarin when used for $\geq 6$ months.

Patients with VTE who had completed $\geq 3$ months of therapy were recruited into either the 'active-control' study, in which dabigatran was compared with warfarin, or the 'placebo-control' study, in which dabigatran was compared with placebo. Dabigatran was noninferior to warfarin in preventing recurrent VTE; $1.8 \%$ of the 1,430 patients treated with dabigatran and $1.3 \%$ of the 1,426 patients treated with warfarin had a VTE during the trial (HR 1.44, 95\% CI 0.78-2.64, $P=0.01$ for noninferiority). Notably, major or clinically relevant bleeding occurred less frequently in patients treated with dabigatran than in patients

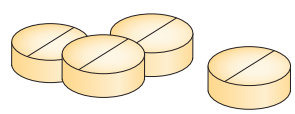
treated with warfarin $(5.6 \%$ versus $10.2 \%$, respectively; HR 0.54 , 95\% CI $0.41-0.71, P<0.001)$.

In the placebo-controlled study, major or clinically relevant bleeding was seen in $5.3 \%$ of the 681 patients treated with dabigatran and in $1.8 \%$ of the 662 patients treated with placebo during the 6-month treatment period (HR 2.92, 95\% CI $1.52-5.60, P=0.001)$. Fewer recurrent VTE events occurred in patients treated with dabigatran than in those treated with placebo for 6 months $(0.4 \%$ versus $5.6 \%$, respectively) or for 12 months (6.9\% versus $10.7 \%$, respectively). Dabigatran could be a safe, convenient, and effective alternative to warfarin for the long-term prevention of recurrent VTE.

Megan Cully

Original article Schulman, S. et al. Extended use of dabigatran, warfarin, or placebo in venous thromboembolism. N. Engl. J. Med. 368, 709-718 (2013) 